\title{
Prognostic value of DKK2 from the Dickkopf family in human breast cancer
}

\author{
YOU-CHENG SHAO ${ }^{1}$, XIAO-CUI NIE ${ }^{2}$, GUO-QING SONG ${ }^{3}$, YAN WEI $^{1}$, PU XIA $^{4}$ and XIAO-YAN XU ${ }^{1}$ \\ ${ }^{1}$ Department of Pathophysiology, College of Basic Medicine Science, China Medical University, Shenyang, Liaoning 110122; \\ ${ }^{2}$ Shenyang Maternity and Infant Hospital, Shenyang, Liaoning 110011 ; ${ }^{3}$ Department of Breast Surgery, \\ Shengjing Hospital of China Medical University, Shenyang, Liaoning 110004; ${ }^{4}$ Department of Cell Biology, \\ College of Basic Medical Science, Jinzhou Medical University, Jinzhou, Liaoning 121001, P.R. China
}

Received May 4, 2018; Accepted September 14, 2018

DOI: $10.3892 /$ ijo.2018.4588

\begin{abstract}
Breast cancer is one of the most frequently diagnosed types of cancer with a high mortality and malignancy rate in women worldwide. The Dickkopf (DKK) protein family, as a canonical Wnt/ $\beta$-catenin pathway antagonist, has been implicated in both physiological and pathological processes. This study aimed to comprehensively characterize the prognostic value and elucidate the mechanisms of DKKs in breast cancer and its subtypes. Firstly, DKK mRNA expression and corresponding outcome were analyzed by means of the Gene Expression-Based Outcome for Breast Cancer Online (GOBO) platform based on PAM50 intrinsic breast cancer subtypes. Subsequently, we extracted breast cancer datasets from the Cancer Genome Atlas (TCGA) and Molecular Taxonomy of Breast Cancer International Consortium (METABRIC) to validate the expression profile and prognostic values from the GOBO platform. Moreover, a protein-protein network was created and functional enrichment was conducted to explore the underlying mechanisms of action of the DKKs. In addition, we uncovered the genetic and epigenetic alterations of DKK2 in breast cancer. The main finding of this study was the differential roles of DKKs in the PAM50 subtypes of breast cancer analyzed. The overall trend was that a high level of DKK2 was associated with a good survival in breast cancer, although it played an opposite role in the Normal-like subtype. We also found that DKK2 carried out its functions through multiple signaling pathways, not limited to the Wnt/ $\beta$-catenin cascade in breast cancer. Finally, we
\end{abstract}

Correspondence to: Dr Xiao-Yan Xu, Department of Pathophysiology, College of Basic Medicine Science, China Medical University, 77 Puhe Road, Shenyang North New Area, Shenyang, Liaoning 110122, P.R. China

E-mail: xyxu@cmu.edu.cn

Key words: Dickkopfs, Dickkopf-related protein 2, breast cancer, bioinformatic analyses, prognosis, gene expression-based outcome for breast cancer online used our own data to validate the bioinformatics analysis data for DKK2 by RT-qPCR. Taken together, our findings suggest that DKK2 may be a potential prognostic biomarker for the Normal-like subtype of breast cancer. However, the prognostic role of DKKs in the subtypes of breast cancer still requires validation by larger sample studies in the future.

\section{Introduction}

For women, breast cancer is the most common type of cancer with a high morbidity rate worldwide (1). According to the data of the National Central Cancer Registry of China (NCCR), 4292,000 newly diagnosed invasive breast cancer cases were reported in 2015 (2). Breast cancer accounts for $15 \%$ of all new-onset malignant tumors in Chinese women (3). The increasing trend of breast cancer reflects the changes in reproductive behavior, the prevalence of obesity and physical inactivity over the past several decades in China (4). Clinical studies have indicated that there is very obvious heterogeneity in breast cancer $(5,6)$. However, the underlying mechanisms related to the heterogeneity of breast cancer have not yet been fully elucidated. Most importantly, a better mechanistic understanding can reveal promising new therapeutic targets. Fortunately, with the development of high-throughput sequencing technologies, the classification of breast cancer extends from pathological types to molecular types. The molecular typing of breast cancer illustrates the heterogeneity of breast cancer in part. Parker et al presented a molecular classification of breast cancer, which was grouped into luminal A (Lum A), luminal B (Lum B), Basal-like, human epidermal growth factor receptor 2-positive $\left(\mathrm{HER}^{+}\right.$), and Normal breast-like subtypes, according to the PAM50 method (7). The PAM50 breast cancer intrinsic classifier has lately been used to assign the molecular subtypes, including Basal, HER2+, Lum A, Lum B and Normal-like subtypes based on the shrunken centroids of gene expression profiles (8). There is no doubt that precision classification can enhance breast cancer diagnosis, treatment and prognosis prediction in clinical practice.

Recently, the massive amount of data generated by the sequencing of tumor samples have spawned a large amount of bioinformatics databases, such as The Cancer Genome Atlas 
(TCGA, https://cancergenome.nih.gov/) and Gene Expression Omnibus (GEO, https://www.ncbi.nlm.nih.gov/geo/), thus enabling us to comprehensively understand tumors from large data analysis at the multiple genetic level. Bioinformatics analysis provides an alternative method with which to explore the genetic function, from DNA to protein. In particular, bioinformatics analysis has an absolute advantage over large quantities of data analysis.

The human Dickkopf (DKK) family members 1,2, 3 and 4 are located within chromosomes $4,8,10$, and 11 (DKK1 maps to 10q11, DKK2 maps to 4q25, DKK3 maps to 11p15.3, and DKK4 maps to 8p11). The DKK family encodes 4 types of secreted protein (DKK1, DKK2, DKK3 and DKK4) that share 2 conserved cysteine-rich domains. The cysteine-rich domain in the $\mathrm{N}$-terminus is unique to each type of DKKs, whereas the other one in the $\mathrm{C}$-terminus is conserved among the family members $(9,10)$. A number of previous studies have demonstrated changes in DKK expression in tumor cell lines or tissues, such as colorectal cancer (11), ovarian carcinoma (12), gastric carcinoma (13) and renal cell carcinoma (14). DKK1 has been shown to be silenced in colon cancer and to be associated with tumorigenesis (11). DKK2 contributes to tumorigenesis in epithelial ovarian carcinoma through the Wnt/ $\beta$-catenin signaling pathway (12). Our group previously found that a decreased DKK3 expression was closely linked to an aggressive behavior and a poor prognosis of gastric cancer (13). The expression of DKK4 has also been shown to be significantly higher in renal cancer tissues compared with adjacent normal kidney tissues (14).

In this study, we adopted cooperatively bioinformatics and experimental methods to performed our analyses. Firstly, we employed the Gene Expression-Based Outcome for Breast Cancer Online (GOBO) platform that developed by Ringnér et al (15) at Lund University Cancer-omics Branch (Lund, Sweden) to perform the comprehensive analysis of DKKs expression profiles and the corresponding prognostic analysis of breast cancer. Secondly, we used our own data to validate the data from bioinformatics analysis on the expression profile of DKK2 in the normal-like subtype compared with normal tissue by means of reverse transcription-quantitative PCR (RT-qPCR). Thirdly, the breast cancer dataset from TCGA, Molecular Taxonomy of Breast Cancer International Consortium (METABRIC, http://molonc.bccrc.ca/aparicio-lab/ research/metabric/) and Kaplan-Meier plotter online platforms were used for the analysis of the expression profiles and prognostic roles of DKKs in breast cancer. Fourthly, a DKK2 protein-protein network was constructed and enrichment analysis was conducted to explore the underlying mechanisms of breast cancer. Finally, DKK2 mutation, copy number variation and methylation were performed to explore the genetic alterations in breast cancer. Taken together, the findings of this study suggest that DKK2 may be a potential prognostic biomarker for the normal-like subtype.

\section{Materials and methods}

DKKs mRNA expression profiles in breast cancer and breast cancer subtypes. DKKs transcripts with molecular subtypes (PAM50) in patients with breast cancer were examined using the GOBO platform, which was developed by Ringnér et al at Lund University Cancer-omics Branch (Lund, Sweden), which consists of three main modules, including gene expression analysis, gene co-expression and sample prediction. It is a publicly available platform, including breast cancer subtyperelated gene expression data and corresponding clinical data from 1,881 subjects with breast cancer based on Affymetrix microarray analysis (http://co.bmc.lu.se/gobo). The breast tumor set from the 1,881 samples comprises 11 public datasets, including Basal $(n=280)$, HER2 ${ }^{+}(n=214)$, Lum A $(n=410)$, Lum $B(n=414)$ and Normal-like $(n=255)$ subtypes according to the breast cancer PAM50 molecular subtype classification (15). The expression analyses of DKKs transcripts in the subtypes and histological grade were performed using one-way analysis of variance (one-way ANOVA). Besides, the DKKs associated with the estrogen receptor (ER)-status were analyzed using the Student's t-test. P-values $<0.05$ were considered to indicate statistically significant differences.

Transcript levels of DKKs and clinical outcomes in breast cancer subtypes. The transcript levels of DKKs associated with breast cancer outcome, together with overall survival (OS) and relapse-free survival (RFS), were analyzed accurately and in detail using the Kaplan-Meier Plotter online platform, which integrates a number of tumor-related microarrays and detailed clinical prognostic information, as previously described (16). DKKs transcripts with molecular subtypes and clinical outcome from patients with breast cancer were analyzed using the GOBO outcome analysis module. The association of clinical outcome with DKK2 gene expression levels in the subgroups of breast cancer was analyzed through OS and RFS as the endpoint and 10 -year censoring. Breast cancer datasets were stratified into 3 quantiles based on DKK2 expression (lower, medium and upper quartile), DKK2_low (log2 expression -5.749 to -0.305), DKK2 medium (-0.305 to 0.502), and DKK2_high (0.502 to 5.422). Subsequently, the Cox proportional hazards model was applied to estimate the hazard ratio, and the ER status, node status, grade, age and tumor size indicator were used as covariates. Logrank P-values are shown as - $\log 10$ (P-value). P-values $<0.05$ were considered to indicate statistically significant differences.

Breast cancer patient specimens and RT-qPCR. We also used our data to further confirm the results of bioinformatics analysis on DKKs and the subtypes. Tissue specimens (17 Normal-like subtype and 28 ER-positive subtype) were obtained from 45 patients (no chemotherapy or radiotherapy prior to resection) at Shengjing Hospital of China Medical University (Shenyang, China) from January, 2011 to September, 2013. Informed written consent was obtained from all participants and the study was approved by Ethics Committee of Shengjing Hospital. Total RNA was extracted from these specimens using the RNeasy Mini kit (Qiagen China, Shanghai, China) according to the manufacturer's instructions. RT-qPCR was carried out using the ABI 7500 RealTime PCR system (Applied Biosystems, Foster City, CA, USA). The RevertAid First Strand cDNA Synthesis kit (Thermo Fisher Scientific, Burlington, ON, Canada) and $\mathrm{SYBR}^{\circledR}$ Premix ExTaq $^{\mathrm{TM}}$ II Kit (Takara Biomedical Technology, Dalian, China) were used. Oligonucleotide primers for DKK2 were used as follows: Forward, 5'-CCCCGTTCATTCCTGTTTG-3' and reverse, 5'-TTCTCCACGGTCCAATCCT-3'. The primers for the internal control, GAPDH, were forward, 5'-CAATGACCCCT TCATTGACC-3' and reverse, 5'-TGGAAGATGGTGATGGG 
ATT-3'. The amplification of DKK2 and GAPDH was performed with 1 cycle at $95^{\circ} \mathrm{C}$ for $10 \mathrm{~min}$ and 40 cycles of $95^{\circ} \mathrm{C}$ for $15 \mathrm{sec}$ and $60^{\circ} \mathrm{C}$ for $60 \mathrm{sec}$. Relative mRNA abundance was normalized to the internal standard, GAPDH, using the $2^{-\triangle \Delta \mathrm{Cq}}$ method (17). The samples were divided into 2 groups at the DKK2 median and classified as high or low DKK2.

Validation of the DKK2 expression profile via TCGA and METABRIC in breast cancer subtypes. The TCGA project, which was developed for cancer research, integrates gene expression profiles, methylation, copy number variation and mutation information in 33 cancer types, which helps us to understand multi-dimensional genetic alterations in tumors (18). TCGA breast cancer dataset (19) is available at the TCGA data portal, such as cBioPortal for Cancer Genomics (http://www.cbioportal.org/) (20). According to the PAM50 classification, TCGA breast cancer dataset (19) was divided into 5 subtypes, including the Basal $(n=98), \operatorname{HER}^{+}(\mathrm{n}=58)$, Lum A $(n=230)$, Lum B $(n=125)$ and Normal-like $(n=8)$ subtypes. Moreover, METABRIC breast cancer dataset covers several gene expression cohorts, and is widely used for the investigation of breast cancer and subtypes. METABRIC breast cancer dataset $(21,22)$ was grouped into the Basal $(n=199)$, HER2 $^{+}(n=220)$, Lum A $(n=679)$, Lum B $(n=461)$ and Normallike $(\mathrm{n}=140)$ subtypes based on the PAM50 classification.

As mentioned above, the GOBO database consists of 11 datasets, which contribute to instability in performance and efficiency of the result. Therefore, we used the publicly available breast cancer dataset with homogeneity and a large sample size to verify the results of GOBO. Subsequently, we undertook breast cancer from TCGA (19) and breast cancer from METABRIC $(21,22)$ to validate expression profile of DKK2 in breast cancer subtypes (22). The expression of DKK2 in the PAM50 subtypes was examined using one-way ANOVA with Dunnett's test with GraphPad prism V7.0 software.P-values $<0.05$ were considered to indicate statistically significant differences.

Validation of the association between DKK2 $\mathrm{mRNA}$ and clinical outcome using the METABRIC database and Kaplan-Meier platform in breast cancer subtypes. We analyzed the survival outcomes of breast cancer and the subtypes according to the differential expression levels of DKK2. Kaplan-Meier survival curves with hazard ratio and logrank P-value were calculated and plotted with the online platform, which is available at Kaplan Meier plotter (http://kmplot.com/analyisis). Kaplan-Meier for breast cancer assessed the relevance of the expression levels of various genes with regards to the clinical outcome (23). Furthermore, we examined METABRIC breast cancer samples $(21,22)$ to validate the clinical outcome of DKK2 in the Normal-like subtype. Data were exported from the cBioPortal platform, and GraphPad prism V7.0 software was then used to perform the survival analysis. The breast cancer sample sets were stratified into 2 quantiles based on DKK2 expression as follows DDK2_medium (medium, 0.424), DKK2_low (-3.04 to 0.424) and DKK2_high (0.424 to 0.534).

Analysis of DKK2 and genes correlation with breast cancer by protein-protein interaction network. To clearly elucidate the underlying mechanisms through which DKK2 is involved in the occurrence and progression of breast cancer at the protein level, we selected breast cancer-related proteins by means of known literature retrieval. Specifically, we conducted the following retrieval expression: 'breast cancer*' and 'gene*' or 'protein*'. Subsequently, we performed PPI network analysis via the Search Tool for the Retrieval of Interacting Genes (STRING v10.5, https://string-db.org/), which provides protein interaction from multiple aspects of evidence, including text-mining, experiments, database, gene co-expression, gene neighborhood, gene-fusion and literature co-occurrence (24). The necessary parameters were set as follows: Meaning of network edges, confidence; minimum required interaction score, medium confidence (0.400). After the PPI network was constructed, Cytoscape software (v3.1.6) was used to visualize the network (25).

Functional and pathway enrichment analysis. Gene ontology (GO) and pathway analysis were performed using the functional enrichment analysis tool (FunRich v3.1.3) software (http://www.funrich.org/), which classifies the biological functions, as well as the signaling pathway of the various proteins involved in the protein networks via hypergeometric distribution algorithm (26). GO analysis consists of molecular function (GO_MF), biological process (GO_BP) and cellular component (GO_CC) (27). Pathway analysis was integrated from the Kyoto Encyclopedia of Genes and Genomes database (KEGG). A threshold value of 0.05 was established for $\mathrm{P}$-values and false discovery rate (FDR) $(\mathrm{P}<0.05$, FDR <0.05).

COSMIC analysisfor DKK2 mutations. DKK2 mutation analysis was carried out using the Catalog of Somatic Mutations in Cancer database (COSMIC) (https://cancer.sanger.ac.uk/cosmic). The pie charts present detailed information about distribution of mutations types and substitutions on the coding strand in breast cancer. The alteration frequency of DKK2 mRNA in breast cancer was performed using BioPortal. All operations were performed according to the cBioPortal instructions. The database query was based on mutation and altered expression of the DKK2 in TCGA breast cancer dataset (19) and METABRIC breast cancer dataset $(21,22)$.

Analysis of DKK2 mRNA expression with DNA methylation analysis and copy number analysis via cBioPortal database. The analysis of DKK2 mRNA expression with DNA methylation and copy number was conducted using the cBioPortal database. DKK2 copy number was analyzed by GISTIC, which is the tool to identify gene targeted by somatic copy-number alteration. Moreover, DKK 2 methylation was analyzed by Human Methylation 450 (HM450) BeadChip kit. cBioPortal has integrated DNA methylation analysis and copy number analysis in a user-friendly manner.

Statistical analyses. The statistical significance of expression of DKKs transcripts in the subtypes was examined by one-way ANOVA. In statistics, Dunnett's test is a multiple comparison method developed by Canadian statistician Charles Dunnett to compare each of a number of treatments with a single control. Multiple comparisons to a control are also referred to as many-to-one comparisons. After ANOVA, Dunnett's test, a post hoc test, was used to perform multiple comparison test. The association of the DKKs with ER-status and grade was analyzed by the Student's t-test. Survival curves were plotted 
$\mathbf{A}$
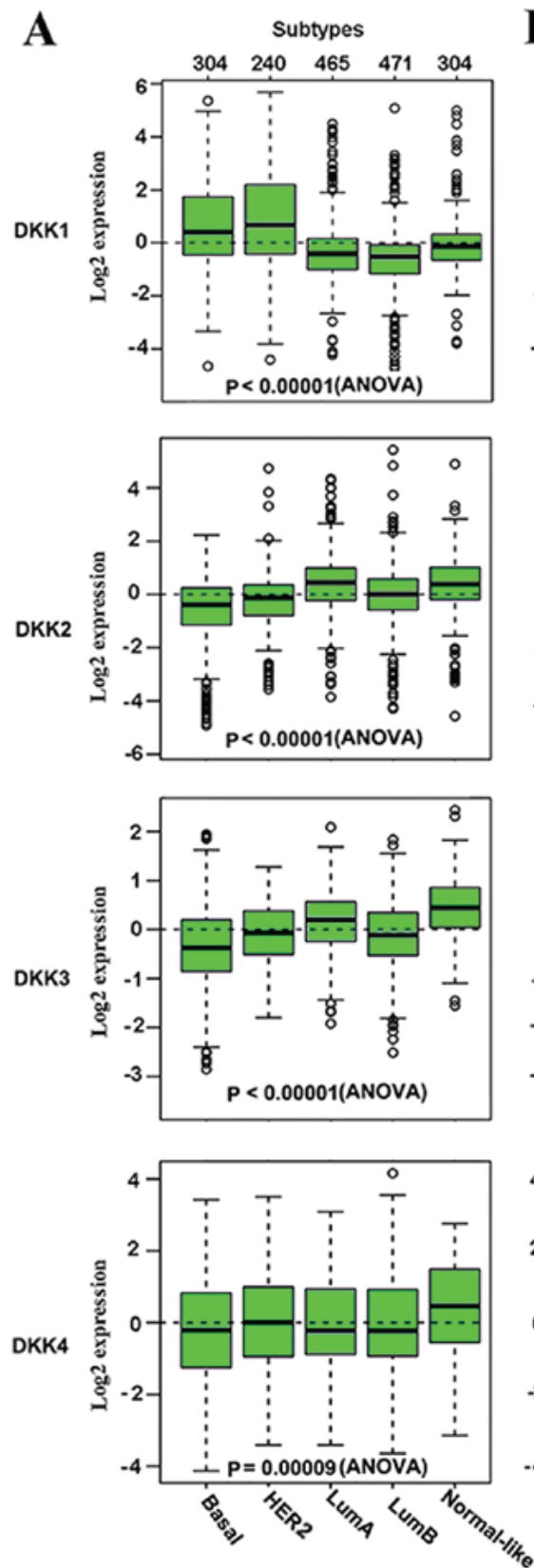
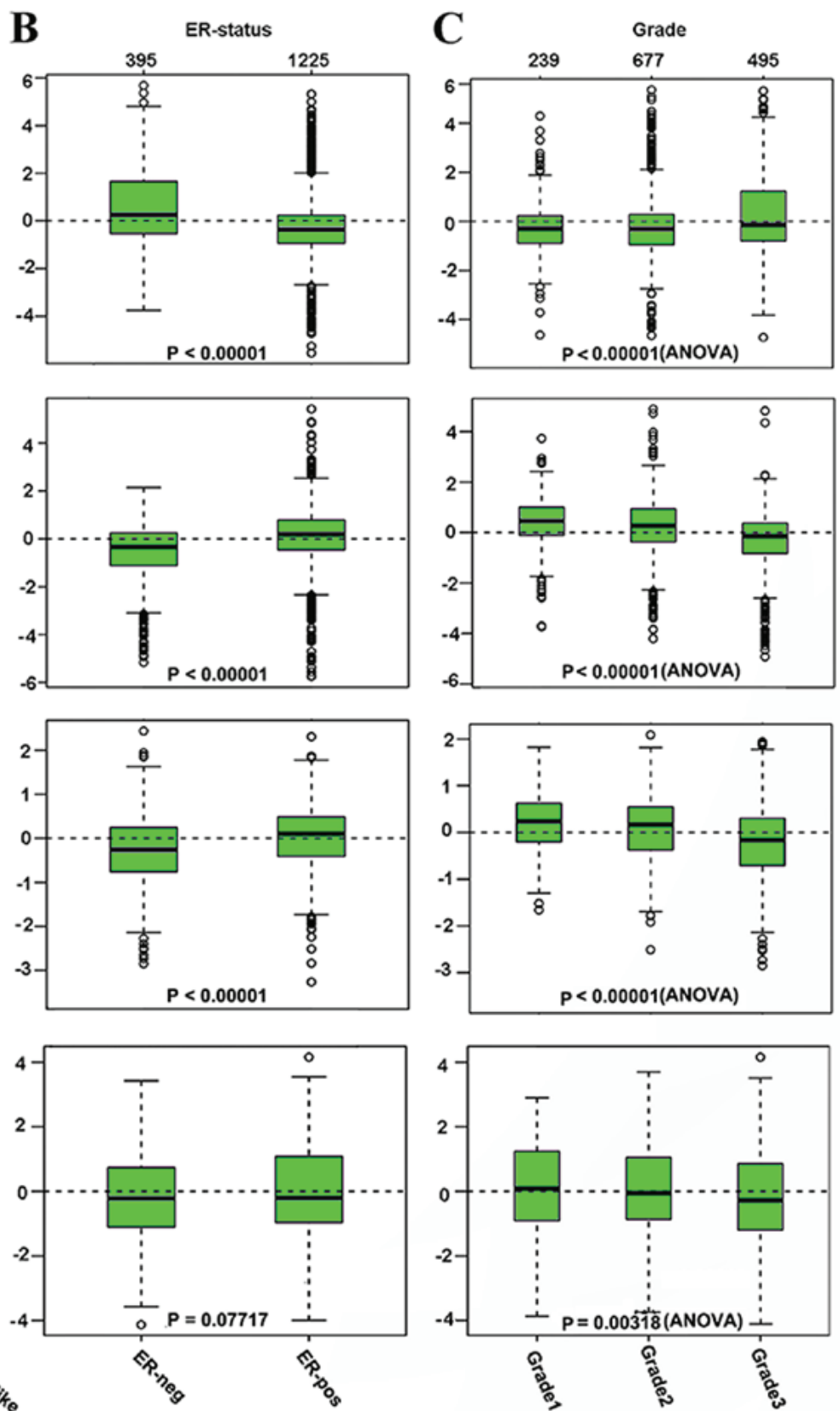

Figure 1. (A) Boxplot of DKKs mRNA expression levels in breast cancer stratified according to PAM50 subtypes. (B) Boxplot of DKKs mRNA expression levels stratified based on the estrogen receptor (ER)-status in breast cancer. (C) Boxplot of DKKs mRNA expression levels for breast cancer stratified by histological grade. The number of patients is shown on top of the bars of in each panel.

using Graphpad Prism software v7.0 (GraphPad Software Inc., San Diego, CA, USA), according to the method of Kaplan and Meier, and the (two-sided) log-rank test was used to assess the statistical significance of differences in OS and RFS among distinct groups of patients. Multivariate analysis of prognostic factors for OS and RFS was performed using the Cox stepwise regression model. P-values $<0.05$ and FDR $<0.05$ were considered to indicate statistically significant differences.

\section{Results}

Expression profiles of DKKs across breast cancer subtypes. Gene expression analysis derived from the GOBO expression module revealed that DKK1 expression was significantly higher in the HER2-enriched, Basal and Normal-like subtype, while it was downregulated in the Lum A and Lum B subtypes $(\mathrm{P}<0.00001$, Fig. 1A). The patients with an ER-negative status exhibited a higher DKK1 expression than those with an ER-positive status $(\mathrm{P}<0.00001$, Fig. 1B). The highest DKK1 transcript expression was observed in Grade 3 tumors, compared with Grade 1 and Grade 2 tumors $(\mathrm{P}<0.00001$, Fig. 1C). The expression of DKK2 and DKK3 was significantly higher in the Lum A and Normal-like subtypes $(\mathrm{P}<0.00001$, Fig. 1A). However, the downregulation of DKK2 and DKK3 was observed in the Basal, HER2 ${ }^{+}$and Lum B subtypes. Unlike DKK1, the patients with an ER-positive status exhibited a higher DKK2 and DKK3 expression than those with an ER-negative status $(\mathrm{P}<0.00001$, Fig. 1B). DKK4 
A

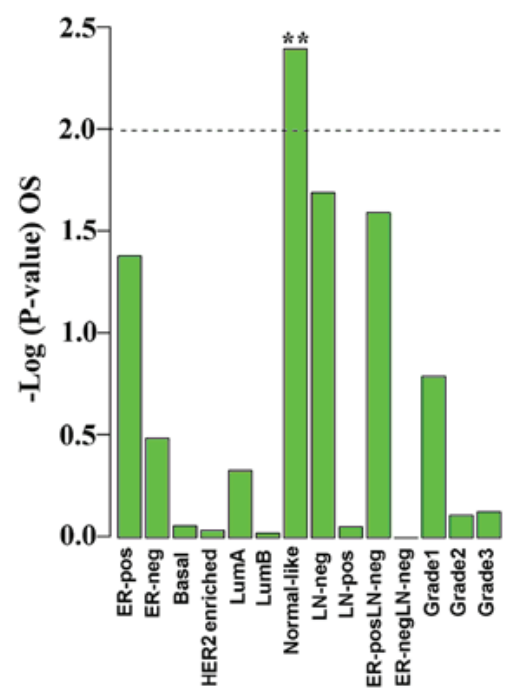

B

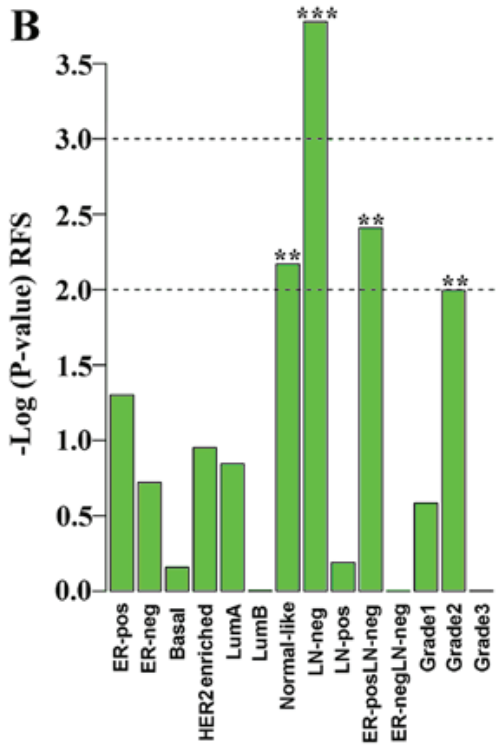

Figure 2. (A) Association of clinical outcome for DKK2 mRNA expression levels was analyzed using overall survival (OS) as the endpoint and 10-year censoring in subgroups of breast cancer. (B) Association analysis of clinical outcome for DKK2 mRNA expression levels was performed using relapse-free survival (RFS) as the endpoint and 10-year censoring in subgroups of breast cancer. Samples in the 1,881-sample set were stratified into 3 quantiles based on DKK2 expression level (lower quartile, -0.305; medium, 0.502; and upper quartile, 5.422) for Kaplan-Meier analysis, including low (log2 expression -5.749 to -0.305), medium (-0.305 to 0.502) and high (0.502 to 5.422) followed by Kaplan-Meier survival analysis in 21 subgroups for 1,379 cases with OS and RFS follow-up. Logrank P-values are shown as $-\log 10$ (P-value). The symbols **, *** on top of the bars stand for $\mathrm{P}<0.01$ and $\mathrm{P}<0.001$, respectively.

expression was higher in the normal-like subtype and lower in the Lum A subtype ( $\mathrm{P}<0.00001$, Fig. 1A). A higher expression level of DKK2, DKK3 and DKK4 was observed in the lower grade and ER-positive tumors $(\mathrm{P}<0.00001$, Fig. 1B). These observations provide further evidence of the DKK1-4 mRNA levels associated with the subtypes, ER status and Grade in breast cancer. Notably, we noted a consistent increase in the expression of DKK family members, with the exception of DKK1, in the normal-like subtypes.

DKKs are associated with the clinical outcome of breast cancer patients. The GOBO outcome module suggested that the DKK2 level was associated with lymph node (LN)-negative $(\mathrm{P}<0.01)$ status, ER-positive/LN-negative status $(\mathrm{P}<0.01)$, Grade $2(\mathrm{P}<0.01)$ and Normal-like subtype $(\mathrm{P}<0.001)$ (Fig. 2). The mRNA expression levels of DKK1, DKK3 and DKK4 were not associated with the clinical prognosis of the breast cancer subtypes (PAM50 classification) and the results were statistically significant. A high DKK2 expression was associated with a good OS of patients with all subtypes of breast cancer $(\mathrm{P}=0.0477$, Fig. 3A). Moreover, a low DKK2 expression was also associated with a poor RFS of patients with all subtypes of breast cancer ( $\mathrm{P}=0.00493$, Fig. 3B). On the contrary, a low DKK2 expression predicted a good OS for patients with the normal-like subtype ( $\mathrm{P}=0.00398$, Fig. 3C). In addition, a low DKK2 expression predicted a good RFS for patients with the normal-like subtype ( $\mathrm{P}=0.00677$, Fig. 3D). On the whole, we comprehensively analyzed the expression profiles and prognostic value of DKKs in breast cancer subtypes. The main data indicated that a high DKK2 mRNA expression was associated with a better prognosis in breast cancer, which is consistent with the findings of a previous study (28). Nevertheless, we focused our attention on the clinical prognostic value of DKK2 in the normal-like subtype.
Validation of the DKK2 expression profile via TCGA and METABRIC in breast cancer subtypes. To verify the accuracy of the expression profiles of DKK2 in the PAM50-based molecular derived from GOBO in subtypes, the TCGA breast cancer dataset (19) and METABRIC breast cancer dataset $(21,22)$ were used to validate the expression profiles of DKK2 in breast cancer subtypes. The METABRIC breast cancer datasets also indicated that the Lum A,Lum B and Normal-like subtypes the expression of DKK2 exhibited an increasing trend (Fig. 4A). Moreover, DKK2 expression was downregulated in the basal and HER2 ${ }^{+}$subtypes ( $\mathrm{P}<0.0001$; Fig. 4A). The TCGA breast cancer dataset (19) also indicated that the transcript level of DKK2 was upregulated in the Lum A, Lum B and normal-like subtypes, and downregulated in the basal and HER2 ${ }^{+}$subtypes $(\mathrm{P}<0.0001)$ (Fig. 4B). These findings are mostly consistent with the results of the GOBO dataset. Moreover, Dunnett's multiple test showed that the expression of DKK2 in Normal-like was significantly higher than Basal and HER2 ${ }^{+}$subtype $(\mathrm{P}<0.0001)$ (Fig. 4A and B). However, there are a few samples for the normal-like subtype in the TCGA dataset, which may account for the fact that the results shown in Fig. 4B are slightly inconsistent with the results shown in Fig. 1A. However, the prognostic role of DKK2 in the subtypes of breast cancer still requires further investigations with larger sample sets in the future.

Validation of the association between DKK2 mRNA expression and clinical outcome via the METABRIC database and Kaplan-Meier platform in breast cancer subtypes. The Kaplan-Meier platform revealed that a high DKK2 expression was a good prognostic factor for OS in the all subtypes (Fig. 4C). Moreover, an increased DKK2 expression was also an excellent factor for RFS (Fig. 4D). METABRIC breast cancer $(21,22)$ indicated that a downregulated DKK2 mRNA expression was associated with a better prognosis than 
A All subtypes

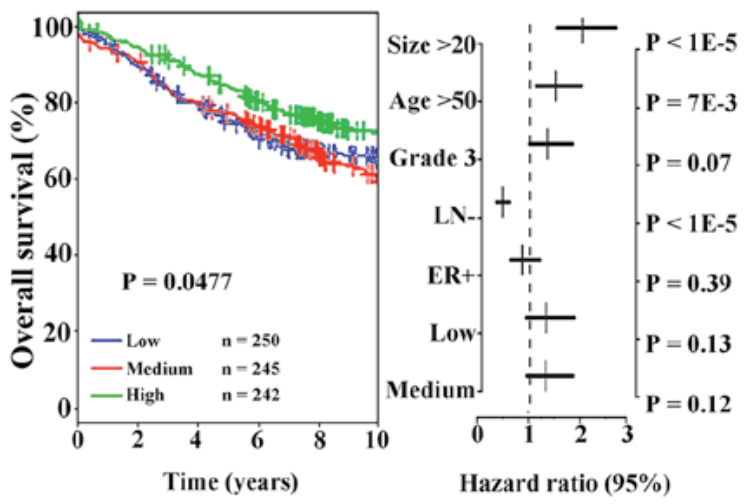

C Normal-like



B All subtypes



D Normal-like

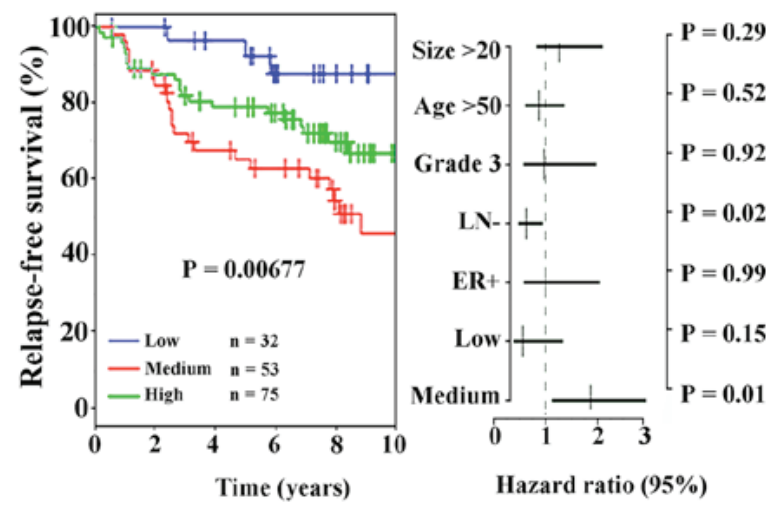

Figure 3. (A) All subtypes were stratified into 3 quantiles based on the DKK2 mRNA level followed by Cox proportional hazards model using lymph node status and stratified histological grade (histological grade 1,2 and 3) and tumor size as covariates, and using overall survival (OS) as the endpoint with 10-year censoring. (B) For all subtypes, the expression of DKK 2 mRNA level was stratified into 3 quantiles based on DKK2 mRNA level followed by Cox multivariable analysis using lymph node status and stratified histological grade (histological grade 1 and 2 vs. 3 ) and tumor size as covariates and using relapse-free survival (RFS) as the endpoint with 10-year censoring. (C) The normal-like subtype was stratified into 3 quantiles based on the DKK2 mRNA level followed by Cox proportional hazards model using lymph node status and stratified histological grade (histological grade 1,2 and 3) and tumor size as covariates, and using OS as the endpoint with 10-year censoring. (D) For the normal-like subtype, the mRNA expression of DKK2 was stratified into 3 quantiles based on the DKK2 mRNA level followed by Cox multivariable analysis using lymph node status and stratified histological grade (histological grade 1 and 2 vs. 3 ) and tumor size as covariates and using RFS as the endpoint with 10-year censoring. Samples in the 1,881-sample set were stratified into 3 quantiles based on the DKK2 expression level (lower quartile, -0.305; medium, 0.502; and upper quartile, 5.422) for Kaplan-Meier analysis, including low (log2 expression -5.749 to $-0.305)$, medium (-0.305 to 0.502$)$, and high (0.502 to 5.422).

the upregulation of its expression in the Normal-like subtype $(\mathrm{P}<0.05$; Fig. 4F). These findings are in line with the results obtained from the GOBO platform.

Evaluation of DKK2 mRNA expression in 45 human breast cancer specimens. RT-qPCR analysis of DKK2 mRNA expression was carried out in the Normal-like subtype and ER-positive subtype of breast cancer. The results revealed that the mRNA level of DKK2 was lower in both types than in the normal tissues $(\mathrm{P}<0.05$, Fig. 4E). Kaplan-Meier analysis revealed that an elevated DKK2 mRNA expression was associated with an unfavorable prognosis of patients with the Normal-like subtype of breast cancer $(\mathrm{P}<0.05$, Fig. $4 \mathrm{G})$. Through our own data validation, our results are consistent with the results obtained from TCGA, METABRIC and GOBO.

PPI network analysis. Based on the information from the literature retrieval, we obtained 39 breast cancer-related proteins, including TP53, ESR1, PGR, ERBB2, CTNNB1, AKT1, APC, ARID1A, ARID1B, ARID2, ASXL1, BAP1, BRCA1, BRCA2,
CASP8, CDH1, CDKN1B, CDKN2A, CCND1, MDM2, ZNF217, MYC, FGFR1, ZNF703, GATA3, KRAS, MAP2K4, MAP3K1, MAP3K13, KMT2C, NCOR1, NF1, PIK3CA, PTEN, SETD2, SMAD4, SMARCD1, STK11 and TBX3. Furthermore, the PPI network consisted of 40 nodes and 76 edges (average node degree of 1.15 and average local clustering co-efficient of 0.313 ) (Fig. 5A). We were aware that DKK2 directly interacted with AXIN1, KRAS, MYC, TP53, CCND1, CDH1, CDKN2A and CTNNB1. The strength of the connection between DKK2 and CTNNB1 was the strongest based on the STRING database. Among these genes, TP53, APC and PTEN are typical tumor suppressor genes (29-31). From the analytical results of PPI network, we also found that DKK2 interacted with SMAD4, MAP3K1 and MAP3K11, which referred to TGF- $3 /$ Smad4 and MAPK signaling pathways through CTNNB1. Additionally, high frequency mutations in the tumor suppressor mentioned genes above have been detected in breast cancer (32). These findings suggest that DKK2 may play a role in multiple signaling pathways. The tumor suppressive effect of DKK2 depends on the synergistic effect of the tumor suppressor genes. 

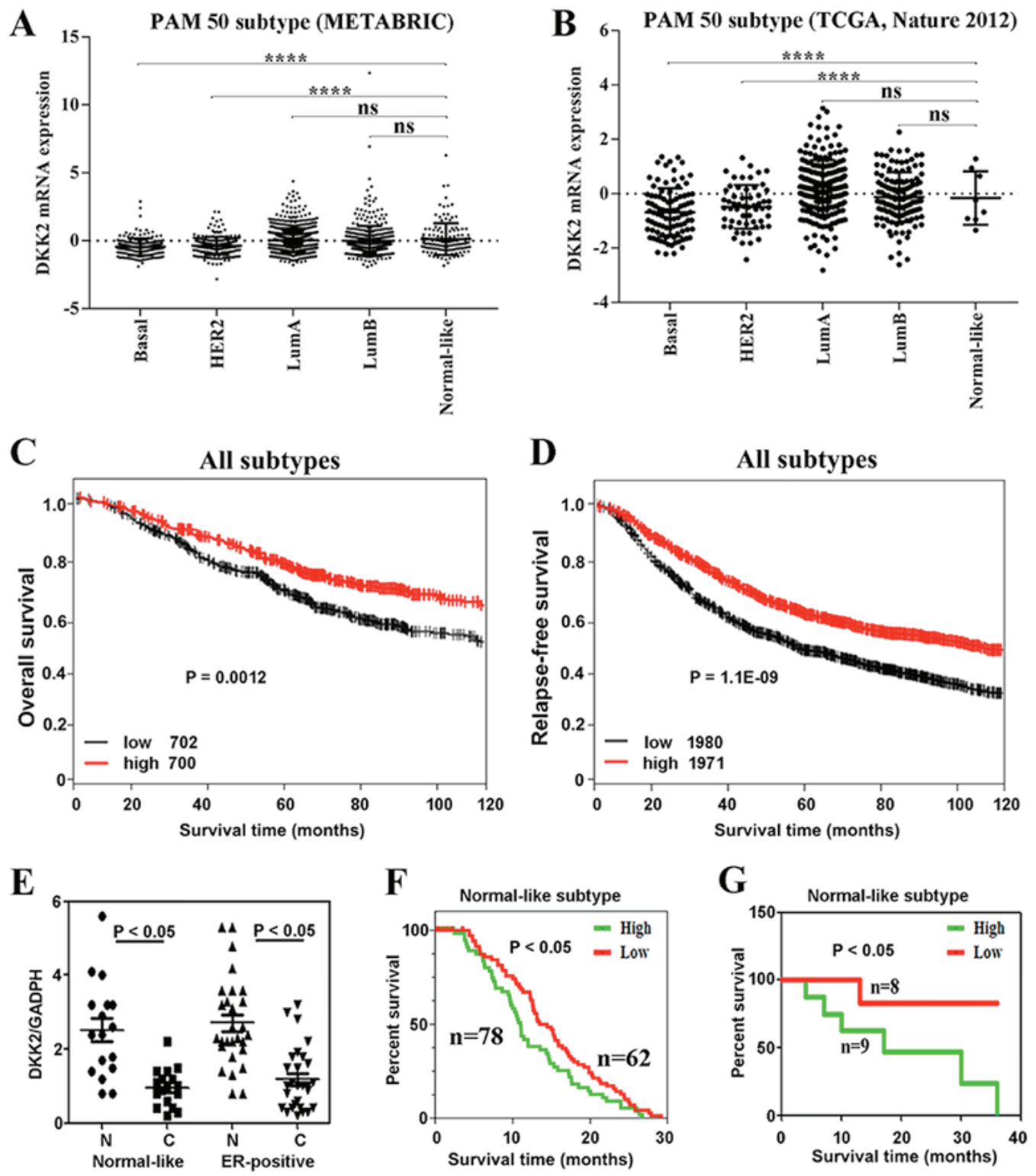

Figure 4. (A and B) METABRIC breast cancer datasets $(21,22)$ and TCGA breast cancer dataset (19) validated the DKK2 expression level in breast cancer subtypes according to PAM50 classification by ANOVA and Dunnett's multiple comparison test. (C and D) Kaplan-Meier online platform validated the association of clinical outcome and DKK2 expression using overall survival (OS) and relapse-free survival (RFS) as endpoints, respectively. The breast cancer sample set was stratified into 2 quantiles based on DKK2 medium (medium, 0.424) expression, including DKK2_low (-3.04 to 0.424) and DKK2_high (0.424 to 0.534). (E) RT-qPCR validated the DKK2 mRNA expression level for normal-like and estrogen receptor (ER ) subtypes versus normal control, respectively. (F and G) METABRIC breast cancer dataset (21,22), TCGA breast cancer dataset $(22,43)$ and our data validated the association of clinical outcome and DKK2 expression using OS, respectively. The symbol ***** and ns on top of the bars stand for $\mathrm{P}<0.0001$ and not significant, respectively;

GOandKEGGpathwayenrichmentanalysis.Tofurtherexplore the protein functions derived from the PPI network, FunRich was used to analyze functional and pathway enrichment. The top significant terms of GO enrichment analysis in FunRich were shown in context. Among these biological process, protein metabolism, transcription, apoptosis, regulation of gene expression, cell communication, signal transduction and regulation of nucleobase. Besides, lipid kinase, cell adhesion, kinase binding, and transcript factor activity were the major molecular function. These proteins were related to several cellular components, including protein complex, cytoplasm, cytosol, nucleoplasm and nucleus (Fig. 5B-D). The top 10 significant terms of pathway enrichment analysis in FunRich were mTOR, PDGFR- $\beta$, EGF-receptor, Class I
PI3K, Arf6 trafficking, LKB1, regulation of CDC42 activity, CDC42, integrin-linked kinase and AP-1 transcription factor network (Fig. 5E). The detailed results are not shown in the text and are available at https://github.com/shaoyoucheng.

COSMIC analysis for DKK2 mutations and cBioPortal analysis for alteration frequency of DKK2. The pie chart illustrated the information of mutations of substitution missense, nonsense, synonymous, insertion and deletion, which were generated by COSMIC. The substitution missense rate was $75 \%$ and the substitution synonymous rate was $25 \%$ of the mutant samples of breast cancer. $\mathrm{G}>\mathrm{A}$ and $\mathrm{C}>\mathrm{A}$ mutation occupied $100 \%$ of the substitution mutation (Fig. 6A and B). The alteration frequency of the DKK2 mutation in breast cancer 
$\mathbf{A}$

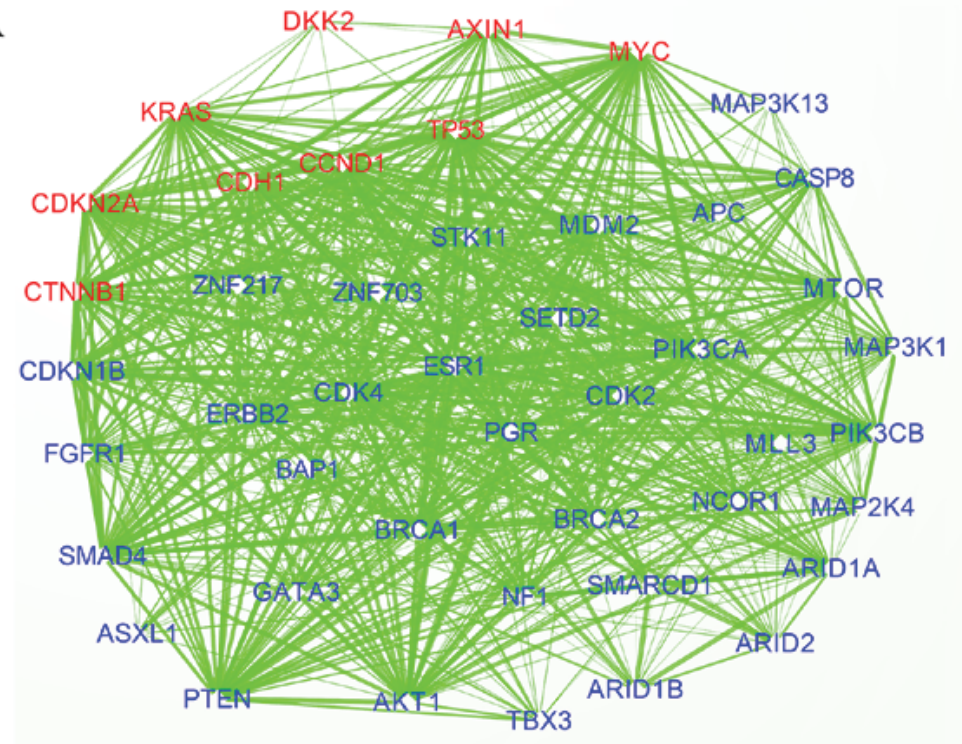

B

-Log 10 (P-value)

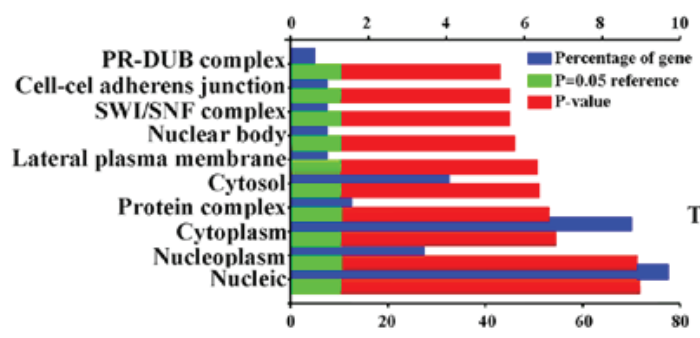

Cellular component

D



C

-Log 10 (P-value)


Figure 5. (A) The protein-protein network for DKK2 in breast cancer was visualized by a Cytoscape v3.6.1. DKK2 and the proteins directly connecting to DKK2 are indicated in red font. Each connection was visualized as a line between the two proteins. The thickness of the line represents the strength of the connection based on the STRING database. (B-E) Gene ontology and pathway analysis were performed for the proteins, which originated from protein-protein network.

was analyzed by BioPortal. Less than $0.1 \%$ of the mutations in the patients with breast cancer were screened (Fig. 6C). Moreover, the event that genetic mutation for DKK2 results in nonsense alteration in the 230th amino acid site was detected in breast cancer (Fig. 6D).

mRNA expression with DNA methylation analysis and copy number analysis. The scatter plot displayed that DKK2 methylation was not associated with the DKK2 mRNA level in breast cancer by the cBioPortal database (Fig. $6 \mathrm{E}$ and F). In addition, the boxplot provided an overview of the DKK 2 mRNA level associated with copy number of deep deletion, shallow deletion, diploid, gain, and amplification, which was generated by the cBioPortal database. Diploid was the cause for the decline in the DKK2 mRNA level in breast cancer (Fig. 6G). On the whole, DKK2 copy number alterations, particularly diploid, account for the elevated DKK2 level in breast cancer.

\section{Discussion}

DKK1 expression has been described to increase early in the development of prostate cancer (33). However, in this study, we confirmed a low DKK1 expression in the patients with grade 1 breast cancer. Xu et al found that an elevated expression of 
A


C

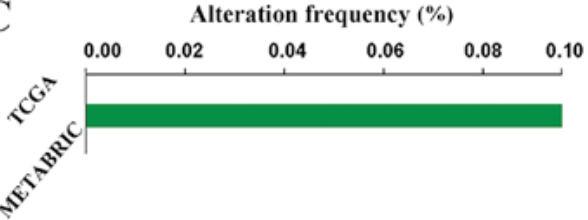

$\mathbf{E}$

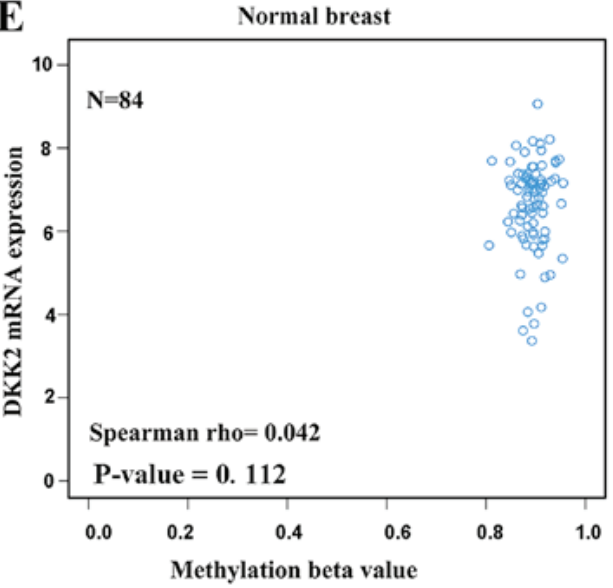

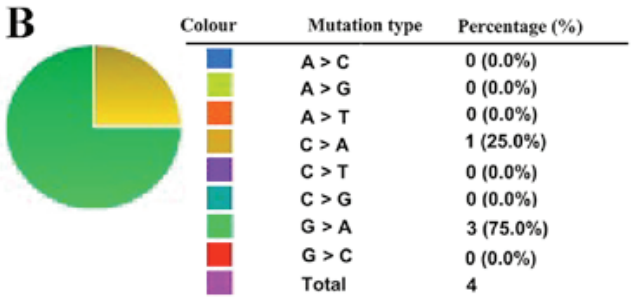
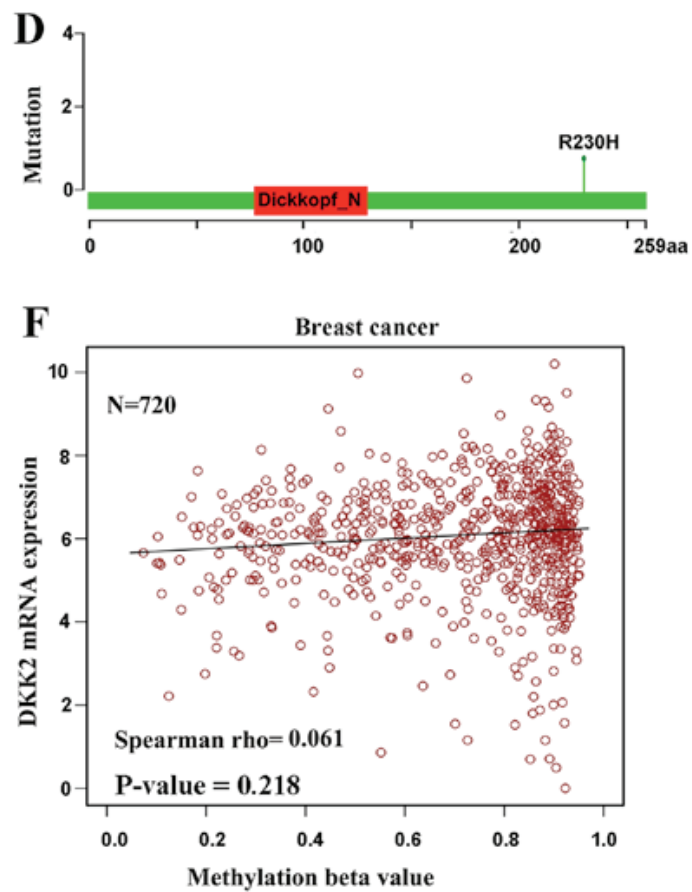

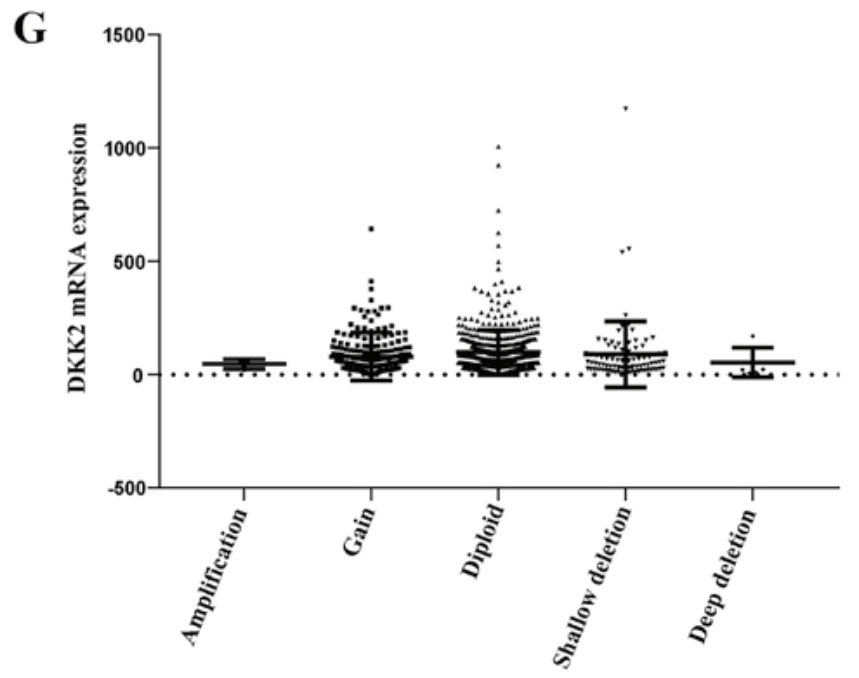

Figure 6. (A and B) Pie-chart showing the distribution and percentage of the mutation type of DKK2 in breast cancer according to COSMIC database. (C and D) Alteration frequency of DKK2 mutations in TCGA breast cancer dataset (19) was analyzed by cBioPortal. (E and F) Correlation analysis between DKK2 mRNA expression and DKK2 methylation level was performed in breast cancer versus normal breast tissue based on cBioPortal by Spearman's correlation analysis, respectively. The regression line was used to illustrate the trend of correlation. (G) The association between DKK2 mRNA expression and DKK 2 copy number variation type was analyzed by cBioPortal in breast cancer.

DKK1 in triple-negative cancers indicated a poor outcome for patients (34). A relative increase in DKK1 expression has also been observed in ER-negative breast cancer patients $(35,36)$. Consistent with the findings of previous studies, in this study, we also confirmed a high DKK1 expression in ER-negative breast cancer.
DKK3 has been reported to be frequently silenced, as a valuable biomarker for breast cancer in the European population $(37,38)$. DKK3 methylation was previously found in $78 \%$ of primary breast cancer tissues in an Asian population (39). Patients harboring DKK3 methylation have been shown to have a poor prognosis in contrast to patients 
retaining an unmethylated DKK3 promoter (37-39). In this study, we found that DKK3 expression was decreased with the progression of tumor stage. Veeck et al found that the downregulation of DKK3 expression predicted a poor OS and short RFS in breast cancer (37). However, the situation is more complex when considering the subtypes of breast cancer. In this study, we found that a high DKK3 expression was associated with a poor Distant metastasis-free survival (DMFS) in ER-positive tumors, ER-positive/LN-negative tumors, and Grade 3 tumors. However, a high DKK3 expression was associated with a good RFS in both ER-negative tumors and LN-positive tumors.

To the best of our knowledge, no previous studies have examined the roles of DKK2 and DKK4 in breast cancer. As shown in this study, we confirmed that DKK2 played significant and various roles in different subtypes of breast cancer. However, we did not find the expression of DKK4 transcripts and corresponding outcomes in breast cancer (data not shown). The differential expression of DKKs may be due to differences in tumor type, tumor stage, or cellular subtypes of breast cancer.

In this study, we focused our attention on the clinical prognostic value of DKKs in breast cancer subtypes (PAM50-based). Our main finding was the different roles of DKK2 in breast cancer and the subtypes. The overall trend was that a high level of DKK2 indicated a poor survival of the Normal-like subtype, while DKK2 was a good indicator for breast cancer (no classification of breast cancer). The TCGA breast cancer dataset (19) and METABRIC breast cancer datasets $(21,22)$ were used to validate the results of expression profile of DKK2 from GOBO database in breast cancer subtypes. We noted that the results shown in Fig. 4A and B were slightly inconsistent with the results shown in Fig. 1A. Furthermore, we found that the landscape of DKK2 expression in breast cancer subtypes was consistent with that of GOBO, excluding the Normal-like subtype. The limitation to a few samples for normal-like subtypes in the TCGA dataset, may account for the above-mentioned results. On the whole, our findings indicated that DKK2 is a potential biomarker in the Normal-like subtype of breast cancer.

PPI analysis suggested that DKK2 carries out its functions through multiple signaling pathways, without being confined to the Wnt/ $\beta$-catenin pathway in breast cancer. Xiao et al proposed that DKK2 secreted by tumor cells acts on cytotoxic lymphocytes, inhibiting STAT5 signaling by impeding STAT5 nuclear localization but independently of the Wnt/ $\beta$-catenin pathway (40). The results from the study by Aravalli et al suggested that DKK2 was completely silenced after MYC-induction in PICM-19-CSCs, suggesting that the absence of DKK2 may be critical for inducing tumorigenesis (41).

Therefore, it can be concluded that DKK2 plays a role in multiple signaling pathways. In addition, we noticed that DKK2 interacted with SMAD4, MAP3K1 and MAP3K11, which are involved in the TGF- $\beta /$ Smad 4 and MAPK signaling pathways through CTNNB1, as illustrated by the PPI network, respectively. Those findings prompted that TGF- $\beta / \mathrm{Smad} 4$ pathway and MAPK pathway were altered after DKK2 acting on CTNNB1, which was consistent with the findings of a previous study (42). Therefore, perhaps the following bold assumption can be made: DKK2 may play a role indirectly through the TGF- $\beta /$ Smad 4 and MAPK pathways.

Furthermore, the genetic alterations in DKK2 in breast cancer are mainly copy number variations and $\mathrm{G}>\mathrm{A}$ substitutions. However, we collected a low number of samples with which to detect other breast cancer types. The prognostic roles of DKKs in the different subtypes of breast cancer warrant further investigations in the future. Experimental studies are required to further explore the role and underlying mechanisms if action of DKK2 in breast cancer.

\section{Acknowledgements}

Not applicable.

\section{Funding}

The present study was funded by National Natural Scientific Foundation of China (no. 81572777), and Shenyang Science and Technology Grant (17-230-9-41).

\section{Availability of data and materials}

The datasets generated and analyzed in the current study are available at TCGA (https://cancergenome.nih.gov/) and cBioPortal for Cancer Genomics (http://www.cbioportal.org/). Additional supplemental results are available at https://github. com/shaoyoucheng.

\section{Authors' contributions}

YCS, XYX, PX and YW designed the study, analyzed the bioinformatics data and drafted the manuscript. XYX, GQS, PX and XCN collected the clinical data and tissue samples, and performed the experiments and the statistical analysis on experiments. All authors reviewed and approved the final manuscript.

\section{Ethics approval and consent to participate}

Human breast cancer samples for our studies were obtained from the Shengjing Hospital of China Medical University. Informed written consent was obtained from all participants and the study was approved by Ethics Committee of Shengjing Hospital.

\section{Patient consent for publication}

Not applicable.

\section{Competing interests}

The authors declare that they have no competing interests.

\section{References}

1. Miller KD, Siegel RL, Lin CC, Mariotto AB, Kramer JL, Rowland JH, Stein KD, Alteri R and Jemal A: Cancer treatment and survivorship statistics, 2016. CA Cancer J Clin 66: 271-289, 2016.

2. Chen W, Zheng R, Baade PD, Zhang S, Zeng H, Bray F, Jemal A, $\mathrm{Yu}$ XQ and He J: Cancer statistics in China, 2015. CA Cancer J Clin 66: 115-132, 2016.

3. Chen JG, Chen HZ, Zhu J, Yang YL, Zhang YH, Huang PX, Chen YS, Zhu CY, Yang LP, Shen K, et al: Cancer survival in patients from a hospital-based cancer registry, China. J Cancer 9: 851-860, 2018.

4. Varghese $\mathrm{C}$ and Shin HR: Strengthening cancer control in China. Lancet Oncol 15: 484-485, 2014. 
5. Song JL, Chen C, Yuan JP and Sun SR: Progress in the clinical detection of heterogeneity in breast cancer. Cancer Med 5: 3475-3488, 2016.

6. Zardavas D, Irrthum A, Swanton C and Piccart M: Clinical management of breast cancer heterogeneity. Nat Rev Clin Oncol 12: 381-394, 2015.

7. Parker JS, Mullins M, Cheang MC, Leung S, Voduc D, Vickery T, Davies S, Fauron C, He X, Hu Z, et al: Supervised risk predictor of breast cancer based on intrinsic subtypes. J Clin Oncol 27: $1160-1167,2009$

8. Tibshirani R, Hastie T, Narasimhan B and Chu G: Diagnosis of multiple cancer types by shrunken centroids of gene expression. Proc Natl Acad Sci USA 99: 6567-6572, 2002.

9. Brott BK and Sokol SY: Regulation of Wnt/LRP signaling by distinct domains of Dickkopf proteins. Mol Cell Biol 22: 6100-6110, 2002.

10. Shao YC, Wei Y, Liu JF and Xu XY: The role of Dickkopf family in cancers: From Bench to Bedside. Am J Cancer Res 7: 1754-1768, 2017

11. Aguilera O, Fraga MF, Ballestar E, Paz MF, Herranz M, Espada J, García JM, Muñoz A, Esteller M and González-Sancho JM: Epigenetic inactivation of the Wnt antagonist DICKKOPF-1 (DKK-1) gene in human colorectal cancer. Oncogene 25 4116-4121, 2006

12. Zhu J, Zhang S, Gu L and Di W: Epigenetic silencing of DKK2 and Wnt signal pathway components in human ovarian carcinoma. Carcinogenesis 33: 2334-2343, 2012.

13. Xu XY, Xia P, Yu M, Nie XC, Yang X, Xing YN, Liu YP, Takano Y and Zheng HC: The roles of REIC gene and its encoding product in gastric carcinoma. Cell Cycle 11: 1414-1431, 2012.

14. Hirata H, Hinoda Y, Majid S, Chen Y, Zaman MS, Ueno K, Nakajima K, Tabatabai ZL, Ishii N and Dahiya R: DICKKOPF-4 activates the noncanonical c-Jun-NH2 kinase signaling pathway while inhibiting the Wnt-canonical pathway in human renal cell carcinoma. Cancer 117: 1649-1660, 2011.

15. Ringnér M, Fredlund E, Häkkinen J, Borg Å and Staaf J: GOBO: Gene expression-based outcome for breast cancer online. PLoS One 6: e17911, 2011.

16. Szász AM, Lánczky A, Nagy Á, Förster S, Hark K, Green JE Boussioutas A, Busuttil R, Szabó A and Győrffy B: Crossvalidation of survival associated biomarkers in gastric cancer using transcriptomic data of 1,065 patients. Oncotarget 7: 49322-49333, 2016

17. Livak KJ and Schmittgen TD: Analysis of relative gene expression data using real-time quantitative PCR and the 2(-Delta Delta C(T)) method. Methods 25: 402-408, 2001.

18. Tomczak K, Czerwińska P and Wiznerowicz M: The Cancer Genome Atlas (TCGA): An immeasurable source of knowledge. Contemp Oncol (Pozn) 19: A68-A77, 2015

19. Koboldt DC, Fulton RS, McLellan MD, Schmidt H, Kalicki-Veizer J, McMichael JF, Fulton LL, Dooling DJ, Ding L, Mardis ER, et al; Cancer Genome Atlas Network: Comprehensive molecular portraits of human breast tumours. Nature 490: 61-70, 2012.

20. Gao J, Aksoy BA, Dogrusoz U, Dresdner G, Gross B, Sumer SO, Sun Y, Jacobsen A, Sinha R, Larsson E, et al: Integrative analysis of complex cancer genomics and clinical profiles using the cBioPortal. Sci Signal 6: pl1, 2013.

21. Curtis C, Shah SP, Chin SF, Turashvili G, Rueda OM, Dunning MJ, Speed D, Lynch AG, Samarajiwa S, Yuan Y, etal; METABRIC Group: The genomic and transcriptomic architecture of 2,000 breast tumours reveals novel subgroups. Nature 486: 346-352, 2012.

22. Pereira B, Chin SF, Rueda OM, Vollan HK, Provenzano E, Bardwell HA, Pugh M, Jones L, Russell R, Sammut SJ, et al: The somatic mutation profiles of 2,433 breast cancers refines their genomic and transcriptomic landscapes. Nat Commun 7 $11479,2016$.

23. Györffy B, Lanczky A, Eklund AC, Denkert C, Budczies J, Li Q and Szallasi Z: An online survival analysis tool to rapidly assess the effect of 22,277 genes on breast cancer prognosis using microarray data of 1,809 patients. Breast Cancer Res Treat 123 725-731, 2010

24. Szklarczyk D, Franceschini A, Wyder S, Forslund K, Heller D, Huerta-Cepas J, Simonovic M, Roth A, Santos A, Tsafou KP, et al: STRING v10: Protein-protein interaction networks, integrated over the tree of life. Nucleic Acids Res 43: D447-D452, 2015 .
25. Agapito G, Guzzi PH and Cannataro M: Visualization of protein interaction networks: Problems and solutions. BMC Bioinformatics 14 (Suppl 1): S1, 2013.

26. Benito-Martin A and Peinado H: FunRich proteomics software analysis, let the fun begin! Proteomics 15: 2555-2556, 2015.

27. The Gene Ontology Consortium: Expansion of the Gene Ontology knowledgebase and resources. Nucleic Acids Res 45: D331-D338, 2017.

28. Mu J, Hui T, Shao B, Li L, Du Z, Lu L, Ye L, Li S, Li Q, Xiao Q, et al: Dickkopf-related protein 2 induces G0/G1 arrest and apoptosis through suppressing $\mathrm{Wnt} / \beta$-catenin signaling and is frequently methylated in breast cancer. Oncotarget 8: 39443-39459, 2017.

29. Duffy MJ, Synnott NC, McGowan PM, Crown J, O'Connor D and Gallagher WM: p53 as a target for the treatment of cancer. Cancer Treat Rev 40: 1153-1160, 2014

30. Xu M, Liu X, Xu Y, Zhu S and Gao Y: Co expression of Axin and APC gene fragments inhibits colorectal cancer cell growth via regulation of the Wnt signaling pathway. Mol Med Rep 16: 3783-3790, 2017

31. Kim DH, Suh J, Surh YJ and Na HK: Regulation of the tumor suppressor PTEN by natural anticancer compounds. Ann NY Acad Sci 1401: 136-149, 2017.

32. Stephens PJ, Tarpey PS, Davies H, Van Loo P, Greenman C, Wedge DC, Nik-Zainal S, Martin S, Varela I, Bignell GR, et al; Oslo Breast Cancer Consortium (OSBREAC): The landscape of cancer genes and mutational processes in breast cancer. Nature 486: 400-404, 2012.

33. Hall CL, Daignault SD, Shah RB, Pienta KJ and Keller ET: Dickkopf-1 expression increases early in prostate cancer development and decreases during progression from primary tumor to metastasis. Prostate 68: 1396-1404, 2008.

34. Xu WH, Liu ZB, Yang C, Qin W and Shao ZM: Expression of dickkopf-1 and beta-catenin related to the prognosis of breast cancer patients with triple negative phenotype. PLoS One 7: e37624, 2012

35. Bu G, Lu W, Liu CC, Selander K, Yoneda T, Hall C, Keller ET and Li Y: Breast cancer-derived Dickkopf1 inhibits osteoblast differentiation and osteoprotegerin expression: Implication for breast cancer osteolytic bone metastases. Int J Cancer 123 1034-1042, 2008

36. Rachner TD, Göbel A, Thiele S, Rauner M, Benad-Mehner P, Hadji P, Bauer T, Muders MH, Baretton GB, Jakob F, et al: Dickkopf-1 is regulated by the mevalonate pathway in breast cancer. Breast Cancer Res 16: R20, 2014.

37. Veeck J, Bektas N, Hartmann A, Kristiansen G, Heindrichs U, Knüchel R and Dahl E: Wnt signalling in human breast cancer: Expression of the putative Wnt inhibitor Dickkopf-3 (DKK3) is frequently suppressed by promoter hypermethylation in mammary tumours. Breast Cancer Res 10: R82, 2008.

38. Kloten V, Becker B, Winner K, Schrauder MG, Fasching PA, Anzeneder T, Veeck J, Hartmann A, Knüchel R and Dahl E: Promoter hypermethylation of the tumor-suppressor genes ITIH5, DKK3, and RASSF1A as novel biomarkers for bloodbased breast cancer screening. Breast Cancer Res 15: R4, 2013.

39. Xiang T, Li L, Yin X, Zhong L, Peng W, Qiu Z, Ren G and Tao Q: Epigenetic silencing of the WNT antagonist Dickkopf 3 disrupts normal Wnt/ $\beta$-catenin signalling and apoptosis regulation in breast cancer cells. J Cell Mol Med 17: 1236-1246, 2013.

40. Xiao Q, Wu J, Wang WJ, Chen S, Zheng Y, Yu X, Meeth K, Sahraei M, Bothwell ALM, Chen L, et al: DKK2 imparts tumor immunity evasion through $\beta$-catenin-independent suppression of cytotoxic immune-cell activation. Nat Med 24: 262-270, 2018.

41. Aravalli RN, Talbot NC and Steer CJ: Gene expression profiling of MYC-driven tumor signatures in porcine liver stem cells by transcriptome sequencing. World J Gastroenterol 21: 2011-2029, 2015.

42. Meng Q, Mongan M, Wang J and Xia Y: Repression of MAP3K1 expression and JNK activity by canonical Wnt signaling. Dev Biol 440: 129-136, 2018

This work is licensed under a Creative Commons Attribution-NonCommercial-NoDerivatives 4.0 International (CC BY-NC-ND 4.0) License. 